\title{
Statistical precision of categorical PATH observations of trunk posture
}

\author{
Jennie Jackson ${ }^{\mathrm{a},{ }^{*}}$, Laura Punnett ${ }^{\mathrm{b}}$ and Svend Erik Mathiassen ${ }^{\mathrm{a}}$ \\ ${ }^{a}$ Centre for Musculoskeletal Research, Department of Occupational and Public Health Sciences, University of \\ Gävle, SE-80176, Sweden ${ }^{b}$ Department of Work Environment, University of Massachusetts Lowell, USA
}

\begin{abstract}
Background: Field studies assessing biomechanical occupational exposures frequently utilize direct observation. PATH (Postures, Activities, Tools, and Handling) is a tool for systematically observing occupational exposures during noncyclic or long, irregular-cycle jobs. While PATH has been used in many studies, its statistical performance under different data collection strategies has not yet been investigated. The purpose of the current study was to examine this issue. Methods: Data from labourers performing the four tasks comprising a 'Jacking Pit Construction' operation was extracted from a previously collected data set. Using a probability based re-sampling bootstrap approach, categorical trunk posture exposure data was compared across nine simulated data collection strategies. Results/Conclusion: At the operational level, dispersion curves showed consistent trends of increased precision with increased sizes of the data set and curves tended to intersect at the expected value seen in the parent data set. At the task level, curves did not always follow the predicted pattern, highlighting the potential pitfalls of using PATH for infrequent tasks and the striking effect that individual workers can have on group exposure estimates of such tasks.
\end{abstract}

Keywords: Work related MSD, exposure assessment, manual materials handling, statistical efficiency, measurement strategies

\section{Introduction}

Field assessment of biomechanical exposures during an occupational task often utilises direct observation of body postures, materials handling demands, and other ergonomic characteristics believed to be indicative of musculoskeletal disorder risks $[3,5,10]$. While observers' judgments may be less precise than instrumented measurements, they have the advantage of being able to characterize multiple exposure dimensions almost simultaneously, and likely at a lower cost, while interfering less in the work process under study $[2,4,11,12]$.

One method for the systematic observation of occupational ergonomic exposures is PATH (Postures, Activities, Tools, and Handling). This tool was developed primarily for the exposure assessment of non-neutral postures and material handling character- istics in non-routinized occupations, i.e., those without short, regular work cycles [1]. Sampling using the PATH approach has mainly been done to obtain group-level data, for example, mean exposures by task and operations, both for descriptive purposes and in order to identify prioritized tasks for intervention [9].

The PATH method has been shown to be both reproducible, given adequate observer training [7], and valid, when compared to results from studies using a bioinstrumentation approach [6,8]. Further, in a recent review of observational exposure assessment methods, Takala and co-workers [10] rated PATH as a "thoroughly developed" method with a "systematic and well-designed sampling approach". Thus, previous PATH studies do offer the user some decision support as to the performance of the method in dif-

\footnotetext{
${ }^{*}$ Corresponding author. Centre for Musculoskeletal Research, Department of Occupational and Public Health Sciences, University of Gävle, SE-80176, Gävle, Sweden. Tel.: +46(0)26 64 8293. E-mail: jennie.jackson@hig.se.
} 
ferent occupational settings and when employed for different purposes.

Still, PATH shares the draw-back of most, if not all, observational methods [10]: it does not provide guidance on how to assess the statistical quality of an exposure sample, let alone advice on the number of samples required to obtain sufficient certainty for a specific purpose. This is a serious concern, since an appropriate sampling strategy with known performance is, arguably, at least as important for the interpretation of the resulting exposure data as the quality of the measurement method itself.

The present study used a probabilistic re-sampling approach to examine the statistical performance of PATH under a series of simulated data collection strategies.

\section{Methods}

This study utilized a sub-set of data from a parent data set collected over a ten-year period during a large construction project in Massachusetts. From the nine operations originally observed, the Jacking Pit Construction operation, performed by the trade group labourers was used in the current study. This operation was selected since it offered a high number of samples (over 3000 observations), and it included several work tasks within the operation, each with minimal specialization required of workers performing the tasks. The tasks involved in the operation were: (1) top work, (2) construction pit wall construction, (3) manual excavation and (4) other miscellaneous work. Only data from workers with a minimum of 40 observations was utilized in the simulations, which resulted in a data set of 3103 observations from 10 workers.

PATH samples were collected at one-minute intervals on workers who were randomly selected (with replacement) for observation from the crew of workers performing the same operation. Thus, on each observation day, all workers within a team who had given informed consent had equal probability of being observed. At the moment of collection, each observation included: task and activity being performed; trunk, shoulder and leg postures; weight in hands if handling any material; and, which tool or material was in use [1]. Each construction team was observed over a period of 3 to 14 days, at 3 to 6 hours per day [9]. For the present paper, the primary exposure of interest was trunk posture, collected as a categorical variable with four divisions: neutral (less than $20^{\circ}$ flexion), mild flexion (between $20^{\circ}$ and $45^{\circ}$ ),
Table 1

Outcome variables calculated for each simulated data set.

\begin{tabular}{|c|c|}
\hline \multicolumn{2}{|c|}{ Postural exposure variables for each operation } \\
\hline$E X P$ & $\begin{array}{l}\text { Operation exposure - \% total time spent in each } \\
\text { exposure category; mean across subjects }\end{array}$ \\
\hline$s_{B S^{*}}^{2}$ & $\begin{array}{l}\text { Overall variance among subjects in EXP (includes } \\
\text { variance between and within subjects) }\end{array}$ \\
\hline$M S_{B T}$ & $\begin{array}{l}\text { Mean squares of task exposures - mean value across } \\
\text { tasks of the squared differences between EXP and } \\
\mathrm{EXP}_{\mathrm{T}}\end{array}$ \\
\hline$C_{T}$ & $\begin{array}{l}\text { Contrast in exposure between tasks }\left(M S_{B T} /\left(M S_{B T}+\right.\right. \\
\left.\left.\boldsymbol{s}_{B S-T}\right)\right)\end{array}$ \\
\hline \multicolumn{2}{|c|}{ Time variables for each task within an operation } \\
\hline \%time & $\begin{array}{l}\% \text { of total operation time spent performing each task; } \\
\text { mean across subjects }\end{array}$ \\
\hline$s_{B S-\% t}^{2}$ & eVariance between subjects in $\%$ time for each task \\
\hline \multicolumn{2}{|c|}{ Postural exposure variables for each task within an operation } \\
\hline $\boldsymbol{E X P _ { T }}$ & $\begin{array}{l}\text { Task exposure - \% time spent in each exposure } \\
\text { category for each task; mean across subjects } \\
\text { performing that task }\end{array}$ \\
\hline$s_{B S-T}^{2}$ & $\begin{array}{l}\text { Variance between subjects in } \mathrm{EXP}_{\mathrm{T}}-\text { mean across } \\
\text { tasks; one value per exposure category }\end{array}$ \\
\hline
\end{tabular}

severe flexion (more than $45^{\circ}$ ), and twist (with or without flexion). Trunk posture was selected because non-neutral postures were frequently observed in all of the operations comprising the large construction dataset, although with different relative frequencies [9], and because it is an important risk factor for back disorders.

To investigate the statistical performance of PATH, nine data collection strategies were simulated ranging from 300 to $4500 \mathrm{PATH}$ samples, i.e. simulating data sets ranging from 5 to 75 hours (samples taken on one minute intervals). For each data collection strategy, 5000 simulated data sets were generated using a probability based (weighted) resampling procedure with the following algorithm: (i) a worker was randomly selected from the group of all workers; (ii) a task was determined for that worker using weighted sample randomization based on the probability in the parent data set of that worker performing each of the possible tasks; and (iii) an exposure for that worker performing that task was determined using weighted sample randomization based on the probability in the parent data set of the worker experiencing each of the possible exposure levels when performing that specific task. 

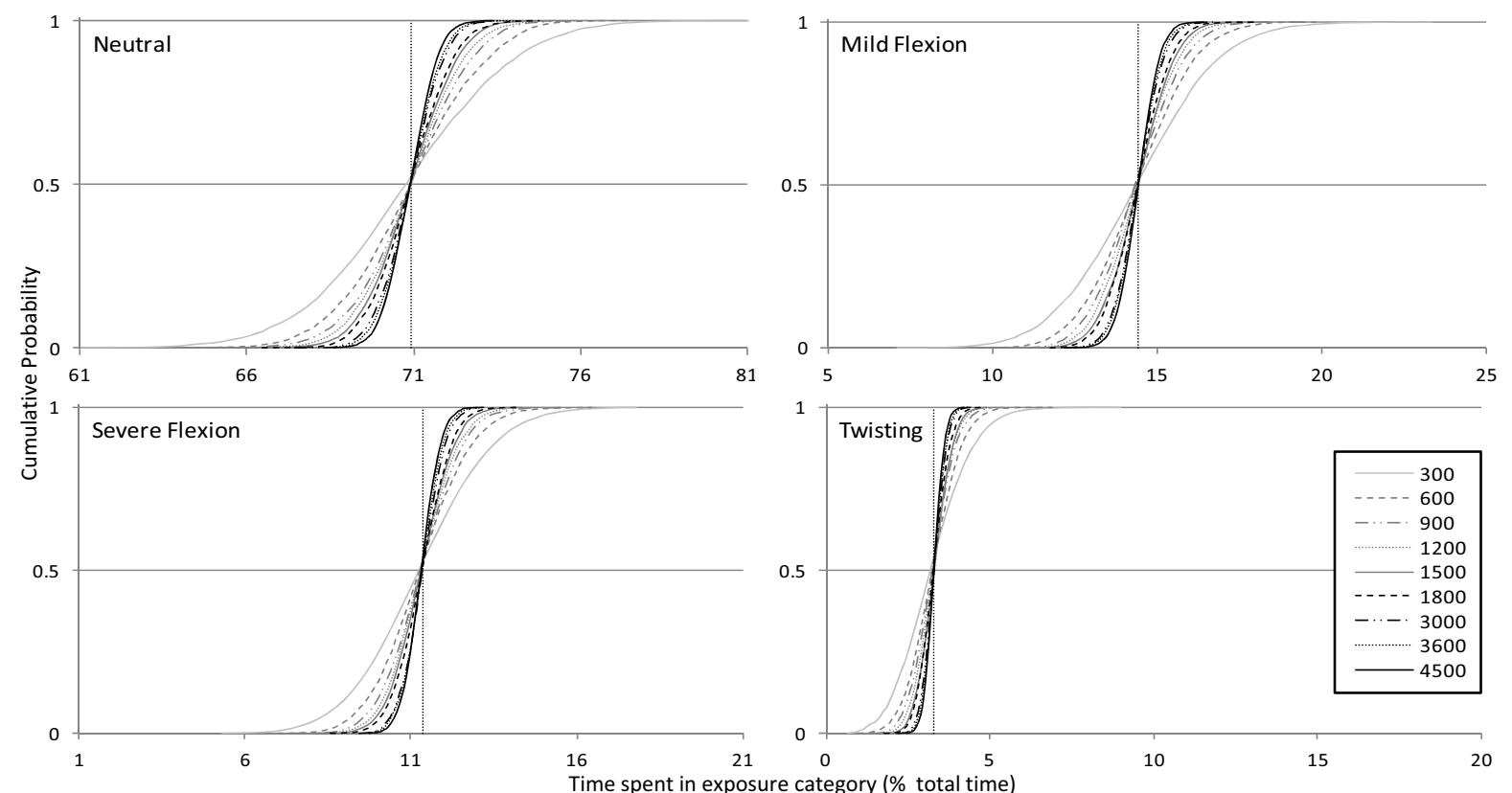

Fig. 1: Cumulative probability by sampling strategies for EXP. Dashed vertical line shows expected value from parent data set.

\section{Results}

At the operation level, dispersion curves showed consistent trends of increased precision with increased size (length) of the data set (figure 1). Dispersion curves tended to intersect at the 'true' value from the parent data set. One exception was data from the one-day data collection strategy, which was often shifted with respect to the other curves. With small samples, dispersion curves for variability data $\left(\mathbf{s}^{2}{ }_{\mathbf{B S}}\right)$ were also biased, showing a shift with respect to the 'true' values as a result of the within-subject variability.

At the task level, the dispersion curves were less predictable and curves from different sampling strategies did not always hold the same basic shape.

\section{Discussion}

The difference in curves at the operation and task levels highlights the potential pitfalls of characterizing infrequent tasks and the striking effect that individual workers can have on group exposure estimates of such tasks. A considerably larger PATH data set is needed if exposures are to be addressed with confidence at a task level than if operations are the level of interest.

\section{References}

[1] B. Buchholz, V. Paquet, L. Punnett, D. Lee and S. Moir, PATH: a work sampling-based approach to ergonomic job analysis for construction and other non-repetitive work, Applied Ergonomics 27 (1996), 177-87.

[2] G.C. David, Ergonomic methods for assessing exposure to risk factors for work-related musculoskeletal disorders, Occupational Medicine 55 (2005), 190-199.

[3] D. Denis, M. Lortie and M. Rossignol, Observation procedures characterizing occupational physical activities: critical review, Int J Occup Saf Ergon 6 (2000), 463-91.

[4] A. Kilbom, Assessment of physical exposure in relation to work-related musculoskeletal disorders--what information can be obtained from systematic observations?, Scandinavian Journal of Work and Environmental Health 20 Spec No (1994), 30-45.

[5] G. Li and P. Buckle, Current techniques for assessing physical exposure to work-related musculoskeletal risks, with emphasis on posture-based methods, Ergonomics 42 (1999), 674-695.

[6] V.L. Paquet, L. Punnett and B. Buchholz, Validity of fixedinterval observations for postural assessment in construction work, Applied Ergonomics 32 (2001), 215-224.

[7] J.-K. Park, J. Boyer, J. Tessler, J. Casey, L. Schemm, R. Gore, L. Punnett, P. Healthy and Safe Employment in Healthcare Project Team, Inter-rater reliability of PATH observations for assessment of ergonomic risk factors in hospital work, Ergonomics 52 (2009), 820 - 829.

[8] S. Tak, L. Punnett, V. Paquet, S. Woskie and B. Buchholz, Estimation of compressive forces on lumbar spine from categorical posture data, Ergonomics 50 (2007), 2082 - 2094.

[9] S. Tak, B. Buchholz, L. Punnett, S. Moir, V. Paquet, S. Fulmer, H. Marucci-Wellman and D. Wegman, Physical ergonomic hazards in highway tunnel construction: Overview from the Construction Occupational Health Program, Applied Ergonomics 42 (2011), 665-671.

[10] E.P. Takala, I. Pehkonen, M. Forsman, G.A. Hansson, S.E. Mathiassen, W.P. Neumann, G. Sjogaard, K.B. Veiersted, R.H. Westgaard and J. Winkel, Systematic evaluation of observational methods assessing biomechanical exposures at work, Scandinavian Journal of Work Environment \& Health 36 (2010), 3-24.

[11] A.J. van der Beek and M.H. Frings-Dresen, Assessment of mechanical exposure in ergonomic epidemiology, Occupational and Environmental Medicine 55 (1998), 291-9.

[12] J. Winkel and S.E. Mathiassen, Assessment of physical work load in epidemiologic studies: concepts, issues and operational considerations, Ergonomics 37 (1994), 979-988. 